\title{
IMPROVED THEORETICAL STELLAR LIBRARY FOR POPULATION SYNTHESIS MODELS
}

\author{
T. LEJEUNE AND R. BUSER \\ Astron. Inst. Univ. Basel, Switzerland
}

We present a standard library for evolutionary and population synthesis studies which provides theoretical stellar energy distributions (SEDs) from UV to IR for the ranges in fundamental parameters, $T_{\text {eff }}(50,000 \mathrm{~K} \sim 2000$ $\mathrm{K}), \log g(-1.0 \sim 5.5)$ and $[M / H](-5.0 \sim+1.0)$. A correction procedure was applied to the original synthetic spectra yielding color-calibrated flux distributions (Lejeune et al. 1997a, 1997b). This stellar library was used to model the integrated light of globular clusters. Comparisons of population models with observed color-magnitude diagrams and integrated spectra of (metal-rich) Bulge globular clusters (Bruzual et al. 1997) show that the semi-empirical flux corrections applied to the original synthetic stellar spectra improve the agreement between model predictions and observations (Fig 1). The population models are also in good agreement with observed $U B V$ and $C M T_{1} T_{2}$ colors of galactic and extragalactic clusters (Lejeune, 1997).

Figure 1. Best fit of the integrated spectrum of NGC6528 (dotted line) from population models using (a) the original SEDs (dashed line) and (b) the color-calibrated SEDs (solid line).

\section{References}

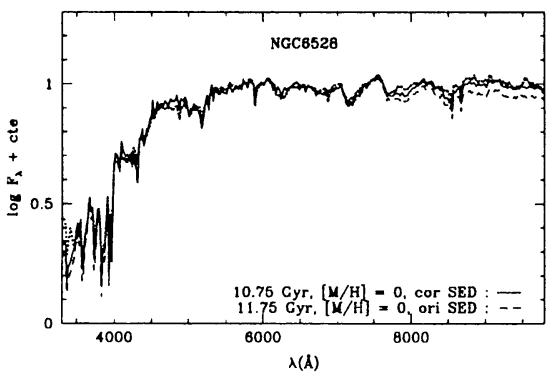

Bruzual, G. et al., 1997, AJ 114, 1531.

Lejeune, T., 1997, PhD thesis, Univ. Louis Pasteur, Strasbourg, France.

Lejeune, T., Cuisinier, F. \& Buser, R., 1997a, A\&AS 125, 229.

Lejeune, T., Cuisinier, F. \& Buser, R., 1997b, A\&AS, in press 